\title{
The Role of Mirena (Intra-uterine progestogens), Other than Contraceptive benefits: Current Concepts and Practices
}

\author{
I BINA
}

Summary:

Mirena is a long acting intrauterine hormone-releasing (LNG-IUS) contraceptive system with a flexible plastic T-shaped frame bearing a levonorgestrel(LNG)containing cylinder which releases small doses levonorgestrel into the uterine cavity after insertion with maximum effect on the endometrium and minimum progestogenic side-effect. LNG causes thinning of the endometrium, atrophy of the endometrial glands and decidualisation of the endometrial stroma. The most common side-effect of LNG-IUS is unscheduled vaginal bleeding in the first 3 months, so proper counseling is needed. In some cases functional ovarian cysts may occur which are relatively small, symptomless and resolved spontaneously within a short time.

Introduction:

Mirena is a long acting intrauterine hormone-releasing contraceptive system. It comprises a small flexible plastic T-shaped frame (length: $3 \mathrm{~mm}$ ) bearing a levonorgestrel(LNG)- containing cylinder. After insertion into the uterus, levonorgestrel-Intrauterine system (LNG IUS) released from the cylinder in small doses (initial release rate, $20 \mathrm{mg} /$ day) into the uterine cavity.

First of all, the concept of intra-uterine administration of progesterone for contraception was introduced in the US in the 1970s. Then, the levonorgestrel-releasing intra-uterine system was devised in Finland gaining a license there for contraception in 1990 and is currently marketed in most European countries, in the UK, since May $1995^{1}$ and in the US since 2000. It is now widely used for its excellent contraceptive benefits. Then the non-contraceptive health benefits of these systems

Address of Correspondence: Dr. Ismatara Bina*, MD, DGO, MCPS, FCPS (O\&G), MRCOG(UK), Consultant Obstetrician and Gynaecologist, Khalishpur Clinic, Plot\#41, Rd.\#101, Khalishpur H/ E, Khulna-9000, Bangladesh

Received: 11 November, 2009

Accepted: 25 May, 2010
Mirena is now licensed for the treatment of menorrhagia in UK because it reduces $97 \%$ of blood loss in 12 months uses. It is also an effective progestogenic endometrial protection in women with Hormone replacement therapy. There are conflicting evidence of LNG-IUS in the management of Fibroids uterus but it reduces fibroid related menstrual blood losses. It has also some beneficial effect in endometriosis and adenomyosis by reducing pain and blood loss. The LNG-IUS also reduces endometrial hyperplasia and may take place in the treatment of eartly endometrial cancer in some cases. The rate of ectopic pregnancy and pelvic inflammatory diseases are reduced in Mirena users compared with IUCD users.

Keywords: Mirena /Intrauterine progestogens/ Noncontraceptive/ LNG-IUS

(J Bangladesh Coll Phys Surg 2010; 28: 167-173)

secondary to the effect of the local action of the progestogen on the endometrium have been observed and researched which has supported the granting of a license for the use of the levonorgestrel-releasing system for the non-contraceptive indication of menorrhagia, specially idiopathic menorrhagia ${ }^{2}$ and the treatment of other endometrial pathology.

Here, we explore the evidence obtained from the use of these intra-uterine hormone delivery systems to provide a review of their current and proposed wider clinical applications, advantages and disadvantages of such devices.

Types of Intrauterine Progesterone

There are four types of Intra-uterine systems: Progestasert, Mirena, Mirena ML and Fibroplant. The latter two are still undergoing clinical trials ${ }^{3}$.

The Mirena Intrauterine System (LNG-IUS) has a Tshaped frame (based on the Nova T IUCD) $32 \mathrm{~mm}$ by $32 \mathrm{~mm}$ made of polyethylene surrounded by an elastomer sleeve in its vertical part. This sleeve is a 1:1 mixture of $52 \mathrm{mg}$ of levonorgestrel and 
polymethylsiloxane. The membrane (also made of polymethylsiloxane) allows a controlled release of 20 mcg of levonorgestrel daily at a constant rate over 5 years $^{3}$. The rate slowly decreases to $15 \mathrm{mcg}$ a day after 5 years and then to $12 \mathrm{mcg}$ at 7 years. Both the serum and intra-uterine levels remain constant over the lifetime of the device in one individual. The serum levels vary from 0.3 to $0.6 \mathrm{mmol} / \mathrm{l}$.

\section{Effects on Endometrium.}

The high levonorgestrel (LNG) concentration in the endometrium down regulate endometrial oestrogen and progesterone receptors, making the endometrium insensitive to circulating $\mathrm{E}_{2}$ (thereby suppressing endometrial growth). After only a couple of months of Mirena use, the glands of endometrium atrophy, the stroma becomes swollen and decidual, the mucosa thins and the epithelium becomes inactive. Vascular changes are thickening of arterial walls, suppression of spiral arterioles and capillary thrombosis. The endometrial changes are uniform within 3 cycles after insertion of the system and no further histological changes take over the long term. Biochemical modulators shows a reduction of cell proliferation and an increase in programmed cell death. These result in a reduction in the endometrial thickness. These changes are reversible and after long-term use; normal menstruation is restored 1month after the removal of the system.

The main principle of non-contraceptive health benefits of the LNG-IUS is based on this endometrial suppression and these include beneficial effects on menorrhagia, as the progestogenic component of combined HRT, in the treatment of hyperplastic and endometrotic endometrium and fibroids and their symptoms. Other health benefits include a reduction in pelvic inflammatory disease and ectopic pregnancy and a possible application in the treatment of premenstrual syndromes. ${ }^{3}$

\section{Effects on ovarian function.}

Over $85 \%$ of women have ovulatory cycles using the LNG-IUS. ${ }^{4}$ and thereafter most cycles are ovulatory. For complete suppression of ovulation, a daily intrauterine release of more than 50mcg of LNG is required. ${ }^{3}$

\section{Benefits}

Role in the management of menorrhagia.

Menorrhagia is experienced by up to $30 \%$ women of reproductive age, ${ }^{3}$ it accounts for $60 \%$ of general practice consultations for menstrual dysfunction, $12 \%$ of gyanecology referrals and is the commonest cause of iron-deficiency anaemia affecting $20-25 \%$ of healthy fertile women in the UK.

One in 20 women aged 30-49 years consult their general practitioner each year with menorrhagia. ${ }^{5}$ Of women referred secondary care, $60 \%$ are likely to have a hysterecomy within 5 years of referral as shown by Coulter et al. ${ }^{6}$ and in most of these women have a normal uterus removed.

In 1993-1994, 73,517 hysterectomies were carried out in England, there was a decline in 1997-1998 when 63,345 operations were carried out. Endometrial ablations had risen markedly from 9945 to 36,440 in the same period.

To date, the management of menorrhagia has relied on pharmacological or surgical therapy. Current pharmaceutical options include non-steroidal antiinflammatory drugs (NSAIDs), antifibrinolytics, danazol, Progestogens and combined oral contraceptives. The surgical treatments include hysterectomy and endometrial ablation or resection.

\section{A. Medical therapies for Menorrhagia}

The LNG-IUS is more effective than oral treatment in the management of menorrhagia. ${ }^{7,8}$ Milsom et al. ${ }^{9}$ studied that Mirena is superior to tranexamic acid and flurbiprofen in reducing blood loss (see Figure-1) on menorrhagia with a lesser side-effects.

Several studies ${ }^{10}$ by various types of drugs showed the reduction of menstrual blood loss by mefenamic acid $25 \%$, Combined oral contraceptive pill $40 \%$, tranexamic acid 50\%, GnRh analogues 75\% and danazol 80\%.

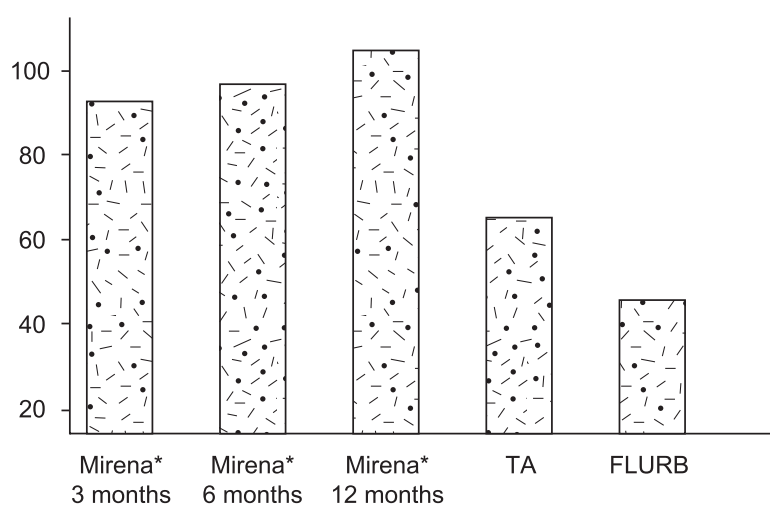

Fig.-1: Reduction in menstrual blood loss as a percentage of mean of two control cycles for Mirena*, Tranexamic acid (TA) and flurbiprofen (FLURB); ${ }^{*} p<0.05$ (between TA and FLURB); ${ }^{* *} P<0.001$ (between Mirena* and TA/FLURB). ${ }^{9}$ 
The study of 20 women with menorrhagia by Andersson and Rubo ${ }^{11}$ used the LNG-IUS and demonstrated a significant reduction in menstrual loss of $85 \%$ at 3 months' and $97 \%$ at 12 months of LNG-I US usage with significant increase in mean serum ferritin by $47 \%$ in the first year of use.( See Table-I, Fig-2 \& Fig-3).

Irvine et al. ${ }^{7}$ showed that Mirena reduced MBL by $94 \%$ after 3 months of treatment (see Table-I), compared with $87 \%$ with oral norethisterone (15 mg daily for 21days in each cycle). More recently, Reid and VirtanenKari ${ }^{12}$ showed that reduction of MBL after 6 months with Mirena was $96 \%$ compared with mefenamic acid was only $17 \%$.

Tang GE, ${ }^{13}$ et al. involved 10 Chinese women with anaemia and who had objectively measured blood loss of $>80 \mathrm{ml}$, used the LNG IUS and demonstrated a reduction of MBL 54\% at one month, $87 \%$ at 3 month and $95 \%$ at 6 month of treatment and an

increase in mean haemoglobin by $19.2 \%$ at 6 months compared with pre-treatment cycles. In addition, Xio et $\mathrm{al}^{14}$ showed that Mirena significantly reduced MBL and increased hemoglobin and ferritin levels over 3 years' follow up.

\section{Table-I}

\section{Summary of comparative and non-comparative studies evaluating the effectiveness of Mirena*} in the treatment of menorrhagia.

\begin{tabular}{|c|c|c|c|c|c|}
\hline \multirow[b]{2}{*}{ Study } & \multirow[b]{2}{*}{$\begin{array}{l}\text { Duration } \\
\text { (months) }\end{array}$} & \multicolumn{2}{|c|}{ Mean menstrual blood loss (ml) } & \multirow[b]{2}{*}{$\begin{array}{c}\text { Reduction in } \\
\text { menstrual blood } \\
\text { loss (\%) }\end{array}$} & \multirow[b]{2}{*}{ Significance } \\
\hline & & Pre-treatment & After treatment & & \\
\hline$\overline{\text { Scholten }^{4}}$ & 12-Jul & 119 & 17 & -86 & $* * *$ \\
\hline \multirow[t]{3}{*}{ Anderssin \& Rybo ${ }^{11}$} & 3 & 176 & 24 & -86 & $* * * *$ \\
\hline & 6 & 176 & 15 & -91 & $* * * *$ \\
\hline & 12 & 176 & 5 & -97 & $* * * *$ \\
\hline \multirow[t]{3}{*}{ Milsaon et al. ${ }^{9}$} & 3 & 203 & 34 & -82 & $* * * *$ \\
\hline & 6 & & 25 & -88 & $* * * *$ \\
\hline & 12 & & 9 & -96 & $* * * *$ \\
\hline \multirow[t]{3}{*}{ Tang \& Lo ${ }^{13}$} & 1 & 183 & 84 & -54 & $* * *$ \\
\hline & 3 & 183 & 24 & -87 & $*$ \\
\hline & 6 & 183 & 10 & -95 & $* * *$ \\
\hline \multirow[t]{4}{*}{ Xiao et al. ${ }^{14}$} & 6 & 124 & 23 & -81 & $* * * *$ \\
\hline & 12 & 124 & 26 & -79 & $* * * *$ \\
\hline & 24 & 124 & 3 & -98 & $* * * *$ \\
\hline & 54 & 124 & 14 & -89 & $* * * *$ \\
\hline \multirow[t]{2}{*}{ Reid \& Virtanen-Kari ${ }^{12}$} & 3 & 122 & 12 & -90 & $* * *$ \\
\hline & 6 & 122 & 5 & -96 & $* * *$ \\
\hline \multirow[t]{2}{*}{ Irvine et al. ${ }^{7}$} & 1 & 105 & 16 & -85 & $* * * *$ \\
\hline & 3 & 105 & 6 & -94 & $* * * *$ \\
\hline
\end{tabular}

* Median values.

$* \mathrm{p}<0.05 ; * * \mathrm{p}<0.01 ; * * * \mathrm{p}<0.005 ; * * * * \mathrm{p}<0.001$. 
Stewart et al. ${ }^{8}$ and Scholten ${ }^{15}$ has also showed the same result in their study: MBL reduced by $86 \%$ (see Table I) with increase of $\mathrm{Hb} \%$ and serum ferritin level (see Figure-2 \& Figure-3). The results of the meta-analysis showed the use of the LNG IUS could significantly reduce menstrual blood loss (range, 74-97\%) in women with confirmed menorrhagia. However, to establish the effectiveness and cost effectiveness relative to other treatments and effect on surgical waiting lists, larger, more powerful, randomised, controlled trails with longer follow-up are required.

The Royal College of Obstetricians and Gynaecologists (RCOG) guideline on the management of menorrhagia in primary care does not identify the LNG-IUS as a treatment option. ${ }^{5}$ However, the RCOG guideline on the management of menorrhagia in secondary care ${ }^{16}$ suggests the LNG-IUS may be used to treat menorrhagia after an assessment of the uterine cavity and endometrial biopsy where appropriate. FFPRHC Guidance ${ }^{1}$ (April 2004) in The LNG-IUS in contraception and reproductive health stated "The LNG-IUS is effective option to treat menorrhagia (Grade A).”

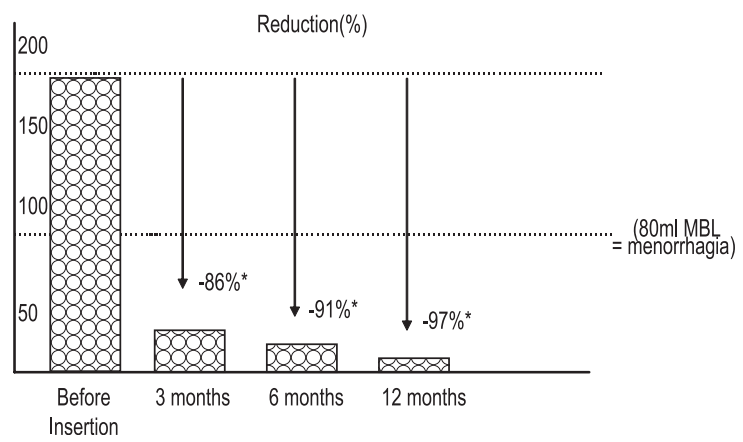

Fig.-2: Reduction in menstrual blood loss (MBL) in women with menorrhagia after 3, 6 and 12 months of Mirena* use; ${ }^{*} p<0.001$ vs baseline. ${ }^{11}$ (a)

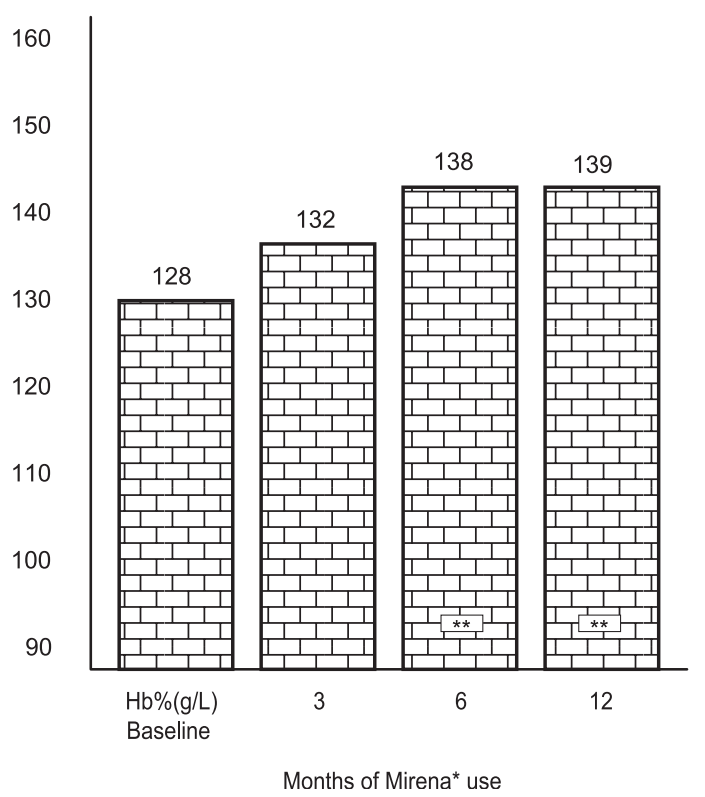

(b)

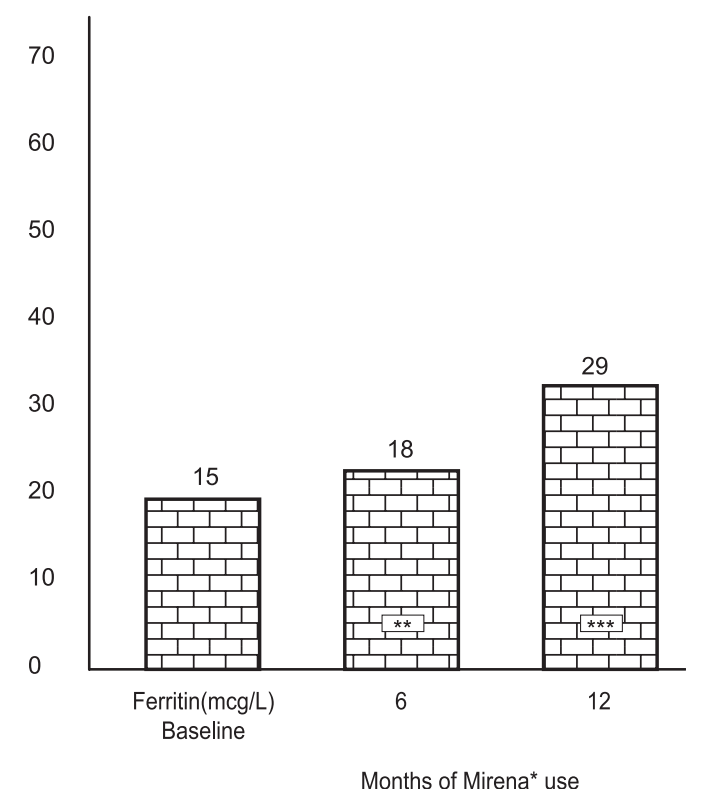

Fig.-3: Mean concentrations ( $+S D)$ of a) hemoglobin and b) serum ferritin in women with menorrhagia before Mirena* insertion and after 3, 6 and 12 months of use (hemoglobin) and 6 and 12 months of use (ferritin); $* * p<0.01, * * * p<0.001$. $^{11}$ 


\section{B. Surgical management for menorrhagia}

A Cochrane review ${ }^{17}$, which included five studies, compared to the LNG-IUS with surgery (hysterectomy, endometrial resection and ablation) and concluded that conservative surgery appeared to be significantly more effective in controlling bleeding at 12 months [odds ratio (OR) 3.99; 95\% CI 1.53-10.38] with beneficial effect in improving quality of life as conservative surgery, in the long term. Reports suggest that the treatment was so effective that $64-82 \%$ of women need not to do hysterectomy and around $14 \%$ of women continue existing medical therapies.

Nagrani and Bowen-Simpkins ${ }^{18}$ showed recently in one study of 4-5-year long-term follow-up of the patients 62 continuation rate of $50 \%$ after a mean 54 months follow-up and only $26.4 \%$ eventually had surgical treatment and an overall $67.4 \%$ avoided surgery.

When MBL is measured using a pictorial assessment chart (PBAC) by Higham et al, ${ }^{19}$ treatment success, defined as a PBAC score of d" 75 at 12 months, has been shown in similar comparisons. In the Visual Analogue Scale (VAS) assessment of the subjective symptoms, sleeping problems were slightly increased in the TCRE group, general feeling of genital health was increased and menstrual pain decreased over time in both the groups.

In a randomized trial by Hurskainen et al. ${ }^{20}$ on quality of life and cost effectiveness of the LNG-IUS $(n=119)$ versus hysterectomy $(n=117)$, for treatment of menorrhagia total cost were 3 folds lower with Mirena than hysterectomy. ${ }^{31}$ Health-related quality of life(HRQoL) and indices of psychosocial well-being improved significantly in both group. Overall Mirena provides effective option for the treatment of menorrhagia with avoidance of the risk associated with a surgical procedure, and without permanent loss of fertility.

The RCOG guideline on management in secondary care $^{16}$ outlines “A progestogen releasing IUD is an effective treatment for reducing heavy menstrual blood loss and should be considered as an alternative to surgical treatment (A).”

FFPRHC Guidance ${ }^{1}$ (April 2004) in the LNG-IUS in contraception and reproductive health stated:

1. "Surgery (hysterectomy, endometrial resection or ablation) is more effective than the LNG-IUS in treating menorrhagia at 1 year (Grade A).”
2. "The LNG-IUS is as effective as conservative surgery (resection and ablation) in the management of menorrhagia after the first year (Grade A).”

3. "Patient satisfaction and quality of life appear similar following LNG-IUS or surgical treatment of menorrhagia (Grade A).”

So the LNG-IUS provides an effective, efficient, welltolerated, cost-effective alternative to other medical and surgical management of menorrhagia.

\section{Progestogenic component of HRT \& Effects on Lipid metabolism}

Hormone replacement therapy (HRT), oestrogen (ERT), is an acceptable option for women who require relief of vasomotor symptoms. Exposure to unopposed oestrogens increases the risk of endometrial hyperplasia and malignancy. Progestogens reduce this risk. Randomized trials suggest that the LNG-IUS is effective in providing endometrial protection from the stimulatory effects of oestrogen, oral ${ }^{21}$ or transdermal. Cohort studies provide evidence of endometrial protection with the LNG-IUS and percutaneous oestradiol ${ }^{22}$ gel use. The majority of postmenopausal women (98.2\%) using an LNG-IUS as the progestogenic component of HRT were amenorrhoeic after 12 months of use. ${ }^{21}$

Mirena causes favourable effects on ERT on the plasma lipid and lipoprotein profiles. A recent study by Raudaskoski et al. ${ }^{23}$ using 2 mg oestradiol valerate and the Mirena intra-uterine system showed HDLcholesterol remaining at baseline level after 12 months of treatment. The LDL-cholesterol levels were reduced by all the LNG-IUS. ${ }^{23}$ These changes might be favorable in cardioprotection.

\section{LNG IUS and endometrosis}

A prospective, non-comparative study showed that of women with the LNG-IUS reported $80 \%$ reduction in primary dysmenorrhoea and MBL. ${ }^{24}$ According to the visual chart devised by Higham et al. ${ }^{19}$, women with endometriosis have a higher baseline mean menstrual score than normal. A pilot study ${ }^{25}$ demonstrated a greatly reduced visual analogue scale for menstrual pain which was associated with a $76 \%$ mean reduction in PBLA chart score.

\section{Fibroids}

The intra-uterine Levonorgestrel systems provide an improvement in fibroid-related menorrhagia with a 
reduction in dymenorrhoea. Five observational studies were identified that investigated the effect of LNG-IUS on uterine fibroids ${ }^{24,25}$ which showed a reduction in MBL and fibroid volume ${ }^{26}$ with LNG-IUS use.

FFPRHC Guidance ${ }^{1}$ (April 2004) in The LNG-IUS in contraception and reproductive health stated "The LNGIUS is effective in the management of menorrhagia, even in the presence of fibroids (Grade C)." "It is not generally recommended that the LNG-IUS be used if fibroids are distorting the uterine cavity (Grade C).” WHOMEC $^{27}$ recommends that if the uterine cavity is distorted with fibroids, the risks of LNG-IUS use outweigh the benefits (WHO 4) because this may not be compatible with insertion.

Endometrial hyperplasia \& Treatment of early endometrial cancer

The LNG-IUS is effective in the treatment of endometrial hyperplasia due to the antiproliferative and suppressive effects on the endometrium. The largest case report $^{28}$ found that all 12 women with simple hyperplasia or atypical hyperplasia had normal endometrium 12 months after LNG-IUS insertion. Montz et al. ${ }^{29}$ showed that intra-uterine progesterone appears to eradicate some cases of presumed stage la grade 1 endometrial cancer in women with a high risk of peri-operative morbidity.

\section{Pelvic inflammatory disease}

A large randomised study ${ }^{30}$ in 5 European countries concluded that women using the LNG IUS had a significantly lower rate of PID than IUCD users. There is also a protective effect in the long term, preventing sexually transmitted infection developing into PID with no protection against sexually transmitted infection.

\section{Prevention of ectopic pregnancy}

The LNG-IUS have a very low failure rates in prevention of pregnancy which makes the ectopic pregnancy rate very low. WHOMEC ${ }^{27}$ recommends that women with a previous ectopic pregnancy may use the LNG-IUS (WHO Category 1: unrestricted use).

\section{Risks}

\section{Ovarian cyst formation}

The incidence of functional ovarian cysts was higher in the LNG-IUS group compared to IUCD users 1.2 versus 0.4 per 100 women-years. ${ }^{9}$ The majority of cysts (94\%) were asymptomatic, relatively small and resolved spontaneously. Occurrence was not related to bleeding pattern, age or FSH levels.

Progestogenic side effects \& Unscheduled vaginal bleeding

Some women do complain of hormonal side effects like oedema, weight gain, headache, breast tenderness, acne and hirsutism and decrease in LDL level. The multicentre contraceptive study in Europe noticed no difference in the weight gain between LNG IUS users and copper IUD users. Irregular vaginal bleeding and spotting in the first few months after insertion is a great problem with LNGIUS. This usually settles within 3-6 months, in which time the full endometrial transformation occurs. 35\% of premenopausal women develop amenorrhoea at the end of the first year of use, and normal menstruation will return once the device is removed.

\section{Conclusions:}

The LNG IUS shows a wider spectrum of benefit other than contraception. Careful pre-insertion counseling; and insertion by a trained fitter can minimize the side effects and bothersome symptoms.

The Mirena is a useful tool in the treatment of menorrhagia and progestogenic compomnent of the hormone replacement armory mainly due to the local effect of Levonorgestrel in the endometrium which may lead to the development of the treatment of other endometrial diseases.

\section{References:}

1. Faculty of Family Planning and Reproductive Health Care (FFPRHC) The levonorgestrel-releasing intrauterine system (LNG-IUS) in contraception and reproductive health FFPRHC Guidance (April 2004) Journal of Family Planning and Reproductive Health Care 2004; 30(2): 99-109

2. Schering Health Care Ltd. Mirena. 0053/0265,1-8.2002. http/ /www.schering.co.uk.

3. Hockey J, Verma V, Panay N, The Wider role of intrauterine progestogens. Progress in Obstetrics and Gynaecology. $1^{\text {st }}$ Edition, London, Elsevier,2005.

4. Scholten PC. The levonorgestrel IUD. Clinical performace and impact on menstruation. Thesis, University of Utrecht, 1989, 94

5. Royal College of Obstetricians and Gynaecologists (RCOG). The Initial Management of Menorrhagia. National EvidenceBased Clinical Guidelines. London, UK: RCOG, 1998.54

6. Coulter A et al. Outcomes of referralsto gynaecology outpatient clinics for menstrual problems: an audit of general practice records. Br J Obstet Gynaecdol 1991; 98: 789-796 
7. Irvine GA et al. Randomised comparative trail of the levonorgestrel intra-uterine system and norethisteronefor treatment of idiopathic menorrhagia. Br J Obstet Gynaecol 1998; 105: 592-598

8. Stewart A et al. The effectiveness of the levonorgestrelreleasing intra-uterine system in menorrhagia: a systematic review. Br J Obstet Gynaecol 2001; 108: 74-86

9. Milsom I, Andersson K, Andersch B, et al. A comparison of flurbiprofen,tranexamic acid, and a levonorgestrel-releasing intrauterine contraceptive device in the treatment of idiopathic menorrhagia. Am J Obstet Gynecol 1991; 164: 879-83.

10. Bonnar J, Sheppard BL. Treatmentof menorrhagia during menstruation: randomised controlled trail of ethamsylate, mefenamic acid, and tranexamic acid. BMJ 1996; 313: 579582

11. Andersson JK, Rybo G. Levonorgestrel-releasing intra-uterine device in the treatment of menorrhagia Br J Obstet Gynaecol 1990; 97: 690-694

12. Reid PC, Virtanen-Kari S. Randomesed comparative trial of the levonorgestrel-releasing intra-uterine system and mefenamic acid for the treatment of idiopathic menorrhagia: a multiple analysis using total menstrual fluid loss, menstrual blood loss and pictorial blood loss assessment charts. Br J Obstet Gynaecol 2005:112:1121-5

13. Tang GE, Lo SS. Levonorgestrel intra-uterine device in the treatment of menorrhagia in Chinese women effecacy versus acceptability. Contraception 1995; 51: 231-235

14. Xiao B, Wu S-C, Chong J, et al. Therapeutic use of the levonorgestrel-releasing intra-uterine system in the treatment of idiopathic menorrhagia. Fertil Steril 2003;79:963-9

15. Scholten PC, Christiaens GCML, Haspels AA,Treatment of menorrhagia by intra-uterine administration of Levonorgestrel. In: Scholten PC. (ed) The levonorgestrel IUD: clinical performance and impact on menstruation (thesis). Utrecht; The Netherlands: Utrecht University Hospital. 1989; 47-55

16. Royal College of Obstetricians and Gynaecologists (RCOG). The Management of Menorrhagia in Secondary Care. National Evidence-Based Clinical Guidelines. London, UK: RCOG, 1999.

17. Majoribanks J, Lethaby A, Farquhar C. Surgery versus medical therapy for heavy menstrual bleeding (Cochrane Review). In: The Cochrane Library, Issue 4, 2003. Chichester, UK: John Wiley \& Sons,Ltd.

18. Nagrani R, Bowen-Simpkins P, Barringto JW. Can the4 levonorgestrel intra-uterine system replace surgical treatment for the management of menorrhagia ? Br J Obstet Gynaecol 2002; 109: 345-347
19. Higham JM, O`Brien PM, Shaw RW. Assessment of menstrual blood loss using a pictorial chart. Br J Obstet Gynaecol 1990; 97: 734-739

20. Hurskainen R et al. Quality of life and cost-effectiveness of levonorgestrel-releasing intra-uterine system vesushysterectomy for treatment of menorrhagia: a randomised trail Lancet 2001: 357: 273-277

21. Raudaskoski T, Tapanainen J, Tomás E, et al. Intrauterine 10 $\mathrm{mg}$ and $20 \mathrm{mg}$ levonorgestrel systems in postmenopausal women receiving oral oestrogen replacement therapy: clinical, endometrial and metabolic response. Br J Obstet Gynaecol 2002; 109: 136-144.

22. Varila E, Wahlström T, Rauramo I. A 5-year follow-up study on the use of a levonorgestrel intrauterine system in women receiving hormone replacement therapy. Fertil Steril 2001; 76: 969-973.

23. Raudaskoski T, Tapanainen E, Tomas E et al. Intrauterine 10 mcg and 20 mcg Levonorgestrel systemsin postmenopausal women receiving oral oesterogen replacement therapy: clinical. endometrial and metabolic. Br J Obstet Gynaecol 2002; 109:136-144

24. Barrington JW, Bowen-Simpkins P. The levonorgestrel intrauterine system in the management of menorrhagia. $\mathrm{Br} \mathrm{J}$ Obstet Gynaecol 1997; 104: 614-616.

25. Vercellini P, Aimi G, Panazza S, De Giorgi O, Pesole A, Crosignani PG. A levonorgestrel - releasing intra-uterine system for the treatment of dysmennorrhea associated with endometriosis. A pilot study. Fertil Steril 1999; 72; 505-508

26. Grigorieva V, Chen-Mok M, Tarasova M, et al. Use of a levonorgestrel- releasing intrauterine system to treat bleeding related to uterine leiomyomas. Fertil Steril 2003; 79: 11941198.

27. World Health Organization (WHO). Medical Eligibility Criteria for Contraceptive Use. Geneva, Switzerland: WHO, 2000.

28. Wildemeersch D, Dhont M. Treatment of non atypical endometrial hyperplasia with a levonorgestrel-releasing intrauterine system. Am J Obstet Gynecol 2003; 188: 12971298.

29. Montz F, Bristow R, Bovicelli A, Tomacruz R, Kuiman R. Intrauterine progesterone treatment of early endometrial cancer. Am J Obstet Gynecol 2002; 186: 651-657

30. Luukainen T, Allonen H, Haukkamma M et al. Effective contraception with levonorgestrel-releasing untra-uterine device. 12 months report of a European multicentre study. Contraception 1987; 36: 169-179 